\title{
Effects of charged defects on the electronic and optical properties of self-assembled quantum dots
}

\author{
Ranber Singh and Gabriel Bester \\ Max-Planck-Institut für Festkörperforschung, Heisenbergstrasse 1, 70569 Stuttgart, Germany.
}

(Dated: November 10, 2018)

\begin{abstract}
We investigate the effects of point charge defects on the single particle electronic structure, emission energies, fine structure splitting and oscillator strengths of excitonic transitions in strained $\mathrm{In}_{0.6} \mathrm{Ga}_{0.4} \mathrm{As} / \mathrm{GaAs}$ and strain-free $\mathrm{GaAs} / \mathrm{Al}_{0.3} \mathrm{Ga}_{0.7} \mathrm{As}$ quantum dots. We find that the charged defects significantly modify the single particle electronic structure and excitonic spectra in both strained and strain-free structures. However, the excitonic fine structure splitting, polarization anisotropy and polarization direction in strained quantum dots remain nearly unaffected, while significant changes are observed for strain-free quantum dots.
\end{abstract}

PACS numbers: 73.21.Hb,42.50.-p,73.21.La,78.67.Hc

\section{INTRODUCTION}

Point charge defects in semiconductors modify their properties, including their electronic structure, trapping and recombination rates for electrons and holes, and luminescence quenching $\frac{1}{1}$. Point defects in semiconductors have been studied extensively with the aim to use their properties for defect engineering. Understanding the properties of point defects is important in studying the electrical and optical properties of semiconductors.

To date, little is known about the influence of point defects on the electronic structure and optical properties of self-assembled semiconductor quantum dots (QDs) grown epitaxially by the Stranski-Krastanow growth mode. These type of QDs provide a quantum system which can be engineered to have a wide range of desired properties ${ }^{2}-\underline{6}$ and provide a rather unique opportunity to investigate various effects known from atomic physics, in a solid state environment $\underline{7,8}$. However, their electronic structure and optical properties can be altered by the presence of defects inside the QDs or in the surrounding barrier ${ }^{9}$. The defects responsible for the electron (hole) trapping in III-V semiconductor heterostructures are the positively (negatively) charged acceptor (donor)-like defects such as vacancies, interstitial or substitutional defects $\underline{10}$. These defects in strained $\operatorname{In}(\mathrm{Ga}) \mathrm{As} / \mathrm{GaAs}$ and strain-free GaAs/Al(Ga)As QDs may develop during the growth process 11,12 . The negatively charged donor-like substitutional Si defect in GaAs QDs are predicted to be stable $\frac{13}{}$. A point charge may also get trapped at a defect site during an optical or electrical measurement. Charged defects inside a QD or in the surrounding region may change the depth and symmetry of the confining potential quite significantly affecting the electronic and optical properties of the QD. The influence of these defects on the optical properties have been shown only in few cases, namely for self-assembled $\mathrm{Ge} / \mathrm{Si}^{14}$, $\mathrm{CdSe} / \mathrm{ZnSe}^{15}$, and InGaAs/AlGaAs ${ }^{16}$ QDs. For colloidal QDs, on the other hand, the phenomena has been extensively investigated. The so-called blinking, is often attributed to the trapping of carriers into defect states on the surface or the surrounding matrix ${ }^{17,18}$ and represents a severe limitation for many potential applications $\frac{19}{}$. For selfassembled QDs, we may speculate that charged defects may be responsible for the large spread of the excitonic fine structure splitting (FSS), polarization direction and polarization anisotropy in strained $\operatorname{In}(\mathrm{Ga})$ As and strainfree $\mathrm{GaAs} / \mathrm{Al}(\mathrm{Ga}) \mathrm{As}_{\mathrm{QDs}}{ }^{20}-23$.

We investigate the effects of point charge defects on the electronic structure, the FSS, emission energies and oscillator strengths of optical transitions in strained $\mathrm{In}_{0.6} \mathrm{Ga}_{0.4} \mathrm{As} / \mathrm{GaAs}$ and strain-free $\mathrm{GaAs} / \mathrm{Al}_{0.3} \mathrm{Ga}_{0.7} \mathrm{As}$ QDs. We use an atomistic empirical pseudopotential description and configuration interaction. The charged defects are modeled at the level of the hydrogenic effective mass description (neglecting central cell effects). We find that the charged defects significantly modify the electronic structure and excitonic spectra in both strained and strain-free QDs. However, the FSS, polarization anisotropy and polarization direction in strained QDs remain nearly unaffected, while in strain-free QDs there are significant changes in these quantities.

\section{COMPUTATIONAL DETAILS}

We consider impurity defects 24 given as, $V=\frac{ \pm 1}{\epsilon\left|\mathbf{r}-\mathbf{r}_{0}\right|}$, following hydrogenic effective mass theory and ignoring the central cell effects 25,26 , where $\epsilon$ is the dielectric constant of the material composing the $\mathrm{QD}$ and $\mathbf{r}_{0}$ is the position of the impurity. The positively $(\oplus)$ and negatively $(\ominus)$ charged point defects are placed at different locations shown schematically in Fig,1 1) inside the QD near its center, 2) in the wetting layer (WL) underneath the base of the QD, 3) inside the QD near the interface with the barrier along the [11̄0] direction. We used a lens-shaped QD with cylindrical base of $25.2 \mathrm{~nm}$ diameter and $3.5 \mathrm{~nm}$ height. We relax the atomic positions within the simulation cell to the minimum strain energy using the valence force field $\operatorname{method} 27,28$. The single particle orbitals and energies of the QD are calculated by using the atomistic empirical pseudopoten- 


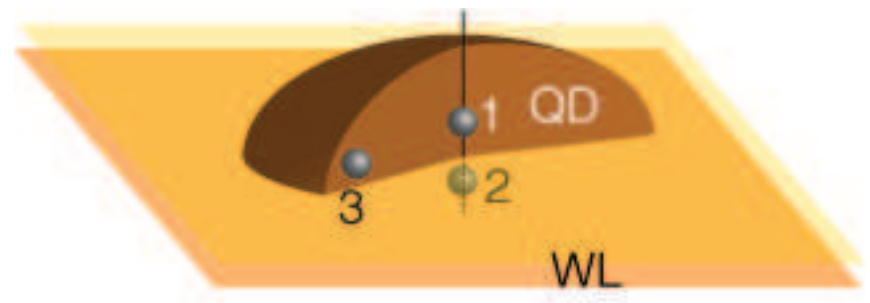

FIG. 1: Position of different point charges; 1) near the QD center, 2) in the WL underneath the base of the QD and 3) near the QD edge along the [110] crystal direction.

tial approach ${ }^{28,29}$, taking strain, band coupling, coupling between different parts of the Brillouin zone and spinorbit coupling into account, retaining the atomistically resolved structure. The Coulomb and exchange integrals are calculated from the atomic wave functions as shown in Ref 30 and the correlated excitonic states are calculated by the configuration interaction (CI) approach ${ }^{31}$. For the CI calculations we use all possible determinants constructed from the twelve lowest energy electron and twelve lowest hole states (spin included), thus accounting for correlations.

\section{RESULTS AND DISCUSSION}

\section{A. Single particle states}

The single particle energies of electron $\left(e_{0,1,2}\right)$ and hole $\left(h_{0,1,2}\right)$ states are given in Figs. 2 and 3 for the different defect configurations in $\mathrm{In}_{0.6} \mathrm{Ga}_{0.4} \mathrm{As} / \mathrm{GaAs}$ and GaAs $/ \mathrm{Al}_{0.3} \mathrm{Ga}_{0.7} \mathrm{As}$ QDs. We observe significant changes in the electronic structure of both strained $\mathrm{In}_{0.6} \mathrm{Ga}_{0.4} \mathrm{As}$ and strain-free GaAs QDs in response to the charged defects. The energetic splitting between $S$ and $P$ states is significantly modified and the energies of electron and hole states blue-shift (red-shift) for negatively (positively) charged defects. This is due to the fact that the negative defects repel (attract) an electron (hole) and accordingly for the positive defects. The energetic splittings between the electron $P$ states $\left(e_{1,2}\right)$ and holes $\left(h_{1,2}\right)$ increase when the defects are placed along the [110] crystallographic direction because it increases the inequivalence between the [110] and [11̄0] directions. In strainfree GaAs QDs there is a larger splitting of the $P$ hole states for the negative defects placed at positions 1 and 2. This is due to the increase of light-hole character in response to the delocalization of the wave functions in z-direction as will be demonstrated next. The decomposition of the S hole state into light-hole (LH) and heavyhole $(\mathrm{HH})$ components is given in Table I. The negative point defects $\ominus$ at positions 1 and 2 lead to an increase of the LH-component, while the positive point defects $\oplus$ at the same positions induce a reduction of the LHcomponent. This can be understood from the reduced and increased Z-component of the hole wave functions.

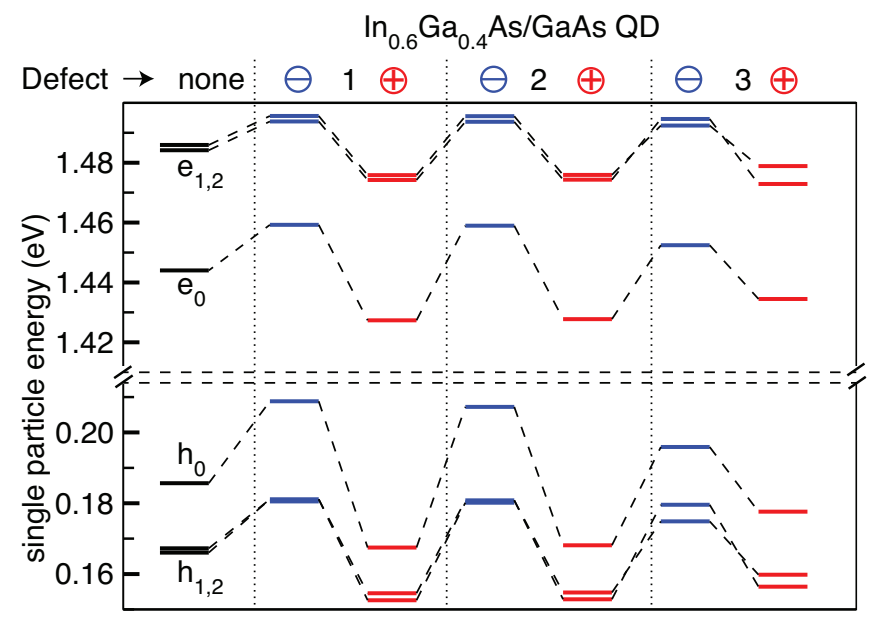

FIG. 2: Single particle energies (relative to the valence band maximum of bulk GaAs) of electron $\left(e_{0}, e_{1,2}\right)$ and hole $\left(h_{0}, h_{1,2}\right)$ states for different point charge defects in $\mathrm{In}_{0.6} \mathrm{Ga}_{0.4} \mathrm{As} / \mathrm{GaAs}$ QDs. The position of the defects $(1,2,3)$ are defined in Fig[1]

The HH is favored when the spread of the wave function in z-direction is small. The introduction of the positive point charge $\oplus$ in the center of the dot leads to a delocalization in-plane and a reduction of the $\mathrm{z}$-character of the wave functions, and hence of its LH-component. The negative point charge $\ominus$ attracts the wave function that becomes more spherical with an increase of its zcharacter, and hence increase the LH-component

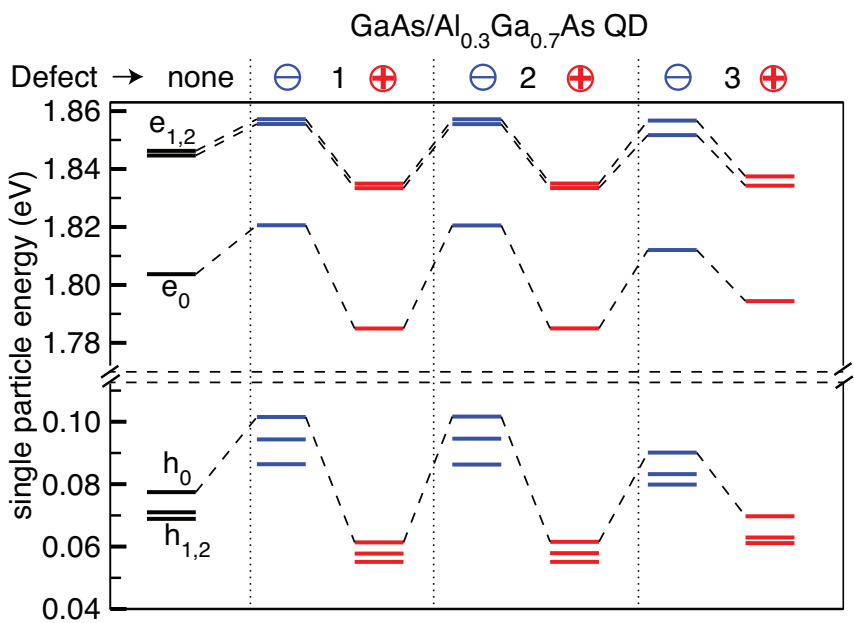

FIG. 3: Single particle energies (relative to the valence band maximum of bulk GaAs) of electron $\left(e_{0}, e_{1,2}\right)$ and hole $\left(h_{0}, h_{1,2}\right)$ states for different point charge defects in $\mathrm{GaAs} / \mathrm{Al}_{0.3} \mathrm{Ga}_{0.7} \mathrm{As}$ QDs. The position of the defects (1, 2, 3) are defined in Fig 1

The isosurfaces enclosing $75 \%$ of the probability density of electron and hole states in strained $\mathrm{In}_{0.6} \mathrm{Ga}_{0.4} \mathrm{As} / \mathrm{GaAs}$ and strain-free GaAs QDs with different point charge configurations are shown in Fig. 4. 
TABLE I: Decomposition of $S$ hole states into $\mathrm{LH}$ and $\mathrm{HH}$ components and Coulomb integrals of hole-hole $\left(\mathrm{J}_{h h}\right)$, holeelectron $\left(\mathrm{J}_{h e}\right)$ and electron-electron $\left(\mathrm{J}_{e e}\right)$ interactions for the $S$ states. The sign '+' ('-') indicate positively (negatively) charged defect. The position of defects $(1,2,3)$ are defined in Fig 1

\begin{tabular}{|c|c|c|c|c|c|c|}
\hline \multirow[b]{2}{*}{ QD } & \multirow[b]{2}{*}{ defect } & \multicolumn{2}{|c|}{$S$ hole state } & \multicolumn{3}{|c|}{ Coulomb integrals } \\
\hline & & $\mathrm{HH}$ & $\mathrm{LH}$ & $\mathrm{J}_{h h}$ & $\mathrm{~J}_{h e}$ & $\mathrm{~J}_{e e}$ \\
\hline \multirow{7}{*}{ GaAs } & none & 89.48 & 09.33 & 25.97 & 24.86 & 23.94 \\
\hline & $1^{\prime}-^{\prime}$ & 84.50 & 13.90 & 35.37 & 26.11 & 22.26 \\
\hline & $2^{\prime-}$ & 84.16 & 14.21 & 35.61 & 26.15 & 22.28 \\
\hline & $3^{\prime}-^{\prime}$ & 84.77 & 13.76 & 29.50 & 20.54 & 24.01 \\
\hline & $1^{\prime}+^{\prime}$ & 90.73 & 08.10 & 20.65 & 22.15 & 25.98 \\
\hline & $2^{\prime}+^{\prime}$ & 90.22 & 08.61 & 20.78 & 22.26 & 25.98 \\
\hline & $3^{\prime}+^{\prime}$ & 88.14 & 10.65 & 26.17 & 23.82 & 23.89 \\
\hline \multirow{7}{*}{$\mathrm{In}_{0.6} \mathrm{Ga}_{0.4} \mathrm{As}$} & none & 94.00 & 02.46 & 24.68 & 20.95 & 18.61 \\
\hline & $1^{\prime}-^{\prime}$ & 92.70 & 03.31 & 29.91 & 21.03 & 17.35 \\
\hline & $2^{\prime-}$ & 92.87 & 03.21 & 29.34 & 21.00 & 17.40 \\
\hline & $3^{\prime-1}$ & 93.90 & 02.52 & 24.52 & 19.95 & 18.64 \\
\hline & $1^{\prime}+^{\prime}$ & 94.34 & 02.25 & 21.13 & 20.12 & 20.05 \\
\hline & $2^{\prime}+^{\prime}$ & 94.36 & 02.23 & 21.26 & 20.29 & 19.97 \\
\hline & $3^{\prime}+^{\prime}$ & 93.86 & 02.56 & 25.10 & 20.30 & 18.67 \\
\hline
\end{tabular}

The defect placed along the [110]0] crystal direction (position 3) breaks the symmetry, which is clearly evident from the spacial distribution of the hole states. The negative defect placed along the [110] crystal direction changes the orientation of the $P$ states as compared to the case of the defect-free QD. The negative (positive) defect at position 3 favors hole (electron) $P$ state along the $[1 \overline{1} 0]$ crystal direction and the effect is so large as to reverse the energetic order of the states. Similar effects are observed for electrons in strain-free $\mathrm{GaAs} / \mathrm{Al}_{0.3} \mathrm{Ga}_{0.7} \mathrm{As}$ QDs (not shown). The spatial extent of the hole states in the GaAs $/ \mathrm{Al}_{0.3} \mathrm{Ga}_{0.7} \mathrm{As} \mathrm{QD}$ deviates significantly from the nearly single-band case of the InGaAs/GaAs QD. This is a consequence of the strong band mixing observed in the GaAs QD and will be further discussed in section IIIC We observe that the negative defects lead to a contraction of the wave functions, while the positive point charges close to the QD center (position 1 and 2) repel the wavefunctions.

The Coulomb integrals for hole-hole $\left(\mathrm{J}_{h h}\right)$, holeelectron $\left(\mathrm{J}_{h e}\right)$ and electron-electron $\left(\mathrm{J}_{e e}\right)$ interactions between the $S$ states are given in Table [. The increase (decrease) in the magnitude of the Coulomb integrals $\mathrm{J}_{h h}$ and $\mathrm{J}_{e e}$ indicates an increase (decrease) in the localization of the hole and electron states, respectively. For the GaAs QD the presence of a negative charge increases the $J_{h h}$ integrals by a large value of about $10 \mathrm{meV}$. The $J_{e e}$ integrals are thereby reduced by only around $1 \mathrm{meV}$. This is related to the rather different effective masses of the electron and the hole in this material. The hole state has an increased ability to localize in the additional Coulomb potential created by the charged defect, compared to the
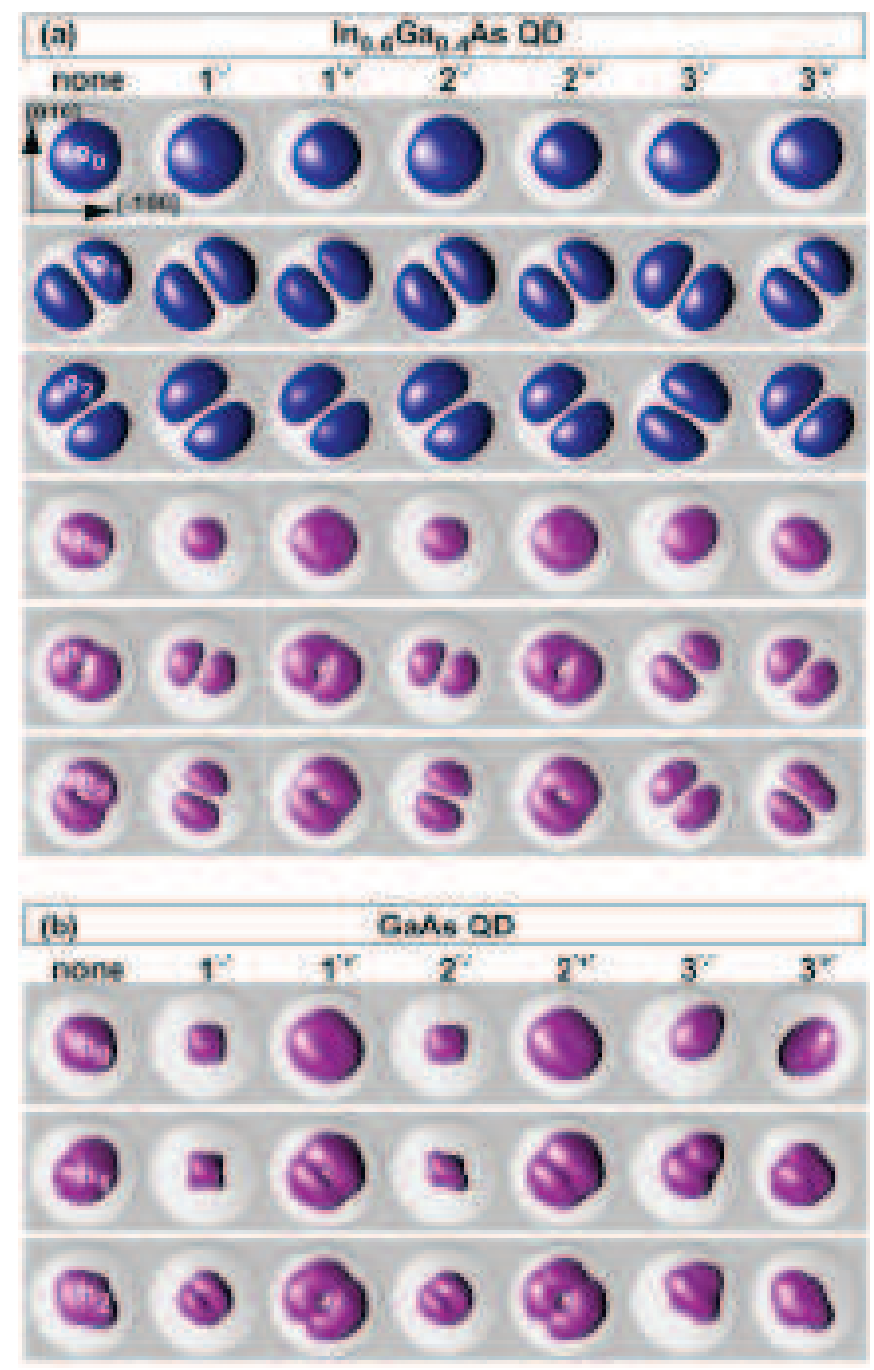

FIG. 4: Square of the wave functions for different point charge defects for $\mathrm{In}_{0.6} \mathrm{Ga}_{0.4} \mathrm{As} / \mathrm{GaAs}$ (a) and $\mathrm{GaAs} / \mathrm{Al}_{0.3} \mathrm{Ga}_{0.7} \mathrm{As}$ (b) QDs. The isosurfaces enclose $75 \%$ of the probability densities. The position of the defects $(1,2,3)$ are defined in Fig 1 .

electron. The changes observed in the $J_{e h}$ Coulomb integrals follow qualitatively the changes observed for the holes, i.e., for a negative defect, the magnitude of integrals increase. The same trends are observed for the InGaAs QD, but with a generally smaller magnitude.

\section{B. Exciton fine structure splitting and polarization}

The emission energy, FSS, polarization direction and polarization anisotropy of neutral excitons in $\mathrm{In}_{0.6} \mathrm{Ga}_{0.4} \mathrm{As} / \mathrm{GaAs}$ and $\mathrm{GaAs} / \mathrm{Al}_{0.3} \mathrm{Ga}_{0.7} \mathrm{As}$ QDs is tabulated in Table II The polarization anisotropy is defined as $\left(\mathrm{I}_{1}-\mathrm{I}_{2}\right) /\left(\mathrm{I}_{1}+\mathrm{I}_{2}\right)$, where $\mathrm{I}_{1}$ and $\mathrm{I}_{2}$ are, respectively, the intensities along major and minor axis of the elliptically polarized total emission of two neutral bright 
TABLE II: Emission energy, FSS, polarization anisotropy and polarization direction with respect to the [110] crystal direction of neutral exciton. The sign ' + ' ('-') indicate positively (negatively) charged defect. The position of the defects $(1,2$, 3) are defined in Fig 1

\begin{tabular}{l|l|cccc}
\hline \hline QD & defect & $\begin{array}{c}\text { energy } \\
(\mathrm{eV})\end{array}$ & $\begin{array}{c}\text { FSS } \\
(\mu \mathrm{eV})\end{array}$ & $\begin{array}{c}\text { polarization } \\
\text { direction }\left(^{\circ}\right)\end{array}$ & $\begin{array}{c}\text { polarization } \\
(\%)\end{array}$ \\
\hline \multirow{4}{*}{ GaAs } & none & 1.700 & 12.2 & 89.8 & 07.5 \\
& $1^{\prime}-^{\prime}$ & 1.692 & 04.4 & 89.5 & 04.5 \\
& $2^{\prime}-^{\prime}$ & 1.692 & 11.9 & 00.1 & 03.5 \\
& $3^{\prime}-^{\prime}$ & 1.699 & 13.9 & 00.1 & 06.5 \\
& $1^{\prime}+^{\prime}$ & 1.700 & 17.1 & 89.9 & 11.9 \\
& $2^{\prime}+^{\prime}$ & 1.700 & 22.7 & 89.9 & 14.9 \\
& $3^{\prime}+^{\prime}$ & 1.699 & 17.9 & 90.0 & 12.2 \\
\hline \multirow{4}{*}{ In $_{0.6} \mathrm{Ga}_{0.4} \mathrm{As}$} & $\mathrm{none}^{\prime}$ & 1.237 & 4.2 & 2.4 & 0.2 \\
& $1^{\prime}-^{\prime}$ & 1.229 & 3.5 & 3.5 & 0.1 \\
& $2^{\prime}-^{\prime}$ & 1.230 & 3.7 & 3.5 & 0.1 \\
& $3^{\prime}{ }^{\prime}+^{\prime}$ & 1.236 & 3.8 & 0.5 & 1.2 \\
& $2^{\prime}+^{\prime}$ & 1.239 & 5.0 & 2.2 & 0.1 \\
& $3^{\prime}+^{\prime}$ & 1.238 & 4.7 & 1.8 & 0.1 \\
\hline \hline
\end{tabular}

exciton transitions. The FSS, polarization anisotropy and polarization direction remain almost unchanged in the presence of charged defects in $\mathrm{In}_{0.6} \mathrm{Ga}_{0.4} \mathrm{As} / \mathrm{GaAs}$ QDs. The polarization direction of the lower exciton is preferably along the [110] direction with small fluctuations in response to the defects. In pure InAs QDs the polarization direction of the lower exciton is along the [110] direction, which remains unaffected in the presence of different defect configurations (not shown). However, there are changes in these quantities in strain-free GaAs $/ \mathrm{Al}_{0.3} \mathrm{Ga}_{0.7}$ As QDs. Polarization anisotropy decrease (increase) for the negative (positive) defects. The envelope functions of electron (hole) states are squeezed (expanded) for the positive defects. The contrary applies to the presence of negative defects (see Fig (4).

The FSS is generally increased in case of positive defects while it is decreased in case of negative defects. This trend follows the observation of the electron-hole direct Coulomb interaction $J_{e h}$ in Table I, where we argued that it is related to the balance between the increase/decrease of electron and hole wave function localization. More explicitly, for negative defects the hole wave function becomes more localized while the electron wave function more delocalized. The effect on the hole overtakes the effect on the electron, and the Coulomb integral has a larger magnitude, compared to the defectfree case. This overall trend is rather intuitive, while the detailed quantitative results are more complicated: For negative defects in the GaAs QD, we obtain a strong reduction of the FSS when going from the defect free case to a defect at position $1(12.2$ to $4.4 \mu \mathrm{eV})$. Moving the defect to position 2 increases the magnitude of the FSS $(11.2 \mu \mathrm{eV})$ but with reversed polarization, so

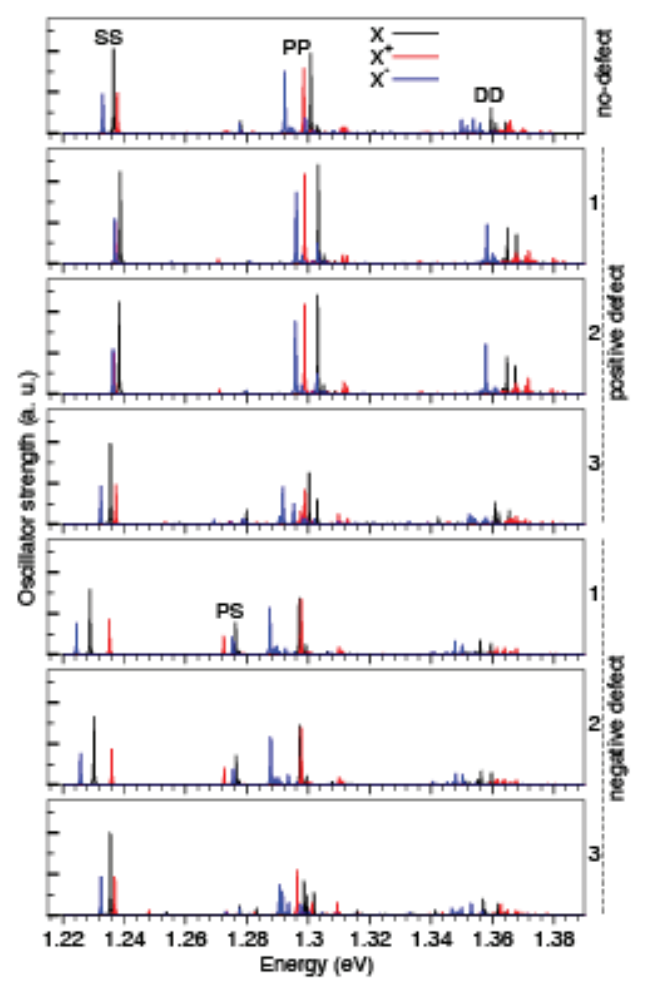

FIG. 5: Absorption spectra of neutral excitons $(\mathrm{X})$ and charged excitons $\left(\mathrm{X}^{+}, \mathrm{X}^{-}\right)$in $\mathrm{In}_{0.6} \mathrm{Ga}_{0.4} \mathrm{As} / \mathrm{GaAs}$ QDs. The positions of the defects are defined $(1,2,3)$ in Fig 1 .

the exciton lines have interchanged (anticrossed ${ }^{32}$ ). We conclude, that the position of the defect along the symmetry axis (through the center of the dot, i.e., the z-axis) and the vertical electric field it creates, has a significant influence on the FSS and is even able to "reverse" it. Strong effects on the vertical electric fields on the FSS

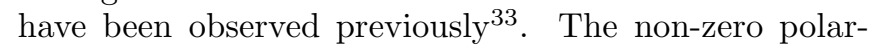
ization anisotropy in GaAs QDs is due to large $\mathrm{LH}-\mathrm{HH}$ mixing. We also notice that the polarization direction in GaAs QDs with cylindrical symmetry is not always along the [110] crystallographic axis. This is due to the atomistic random nature of the barrier composition and hence the interface of the QD.

\section{Excitonic spectra}

The absorption spectra of neutral and charged excitons in InGaAs and GaAs QDs with different point charge defect configurations are given in Fig 5 and 6. The SS, PP and DD labels characterize the different orbital characters of the electron and hole states involved in the optical transition. The actual orbital S, P, D character of the hole wave functions for the GaAs QD is given in Table III. It becomes clear that strong deviations from orbitally pure states exist in GaAs. The states can hardly be classified as "S", "P", "D" as they have a mostly mixed char- 


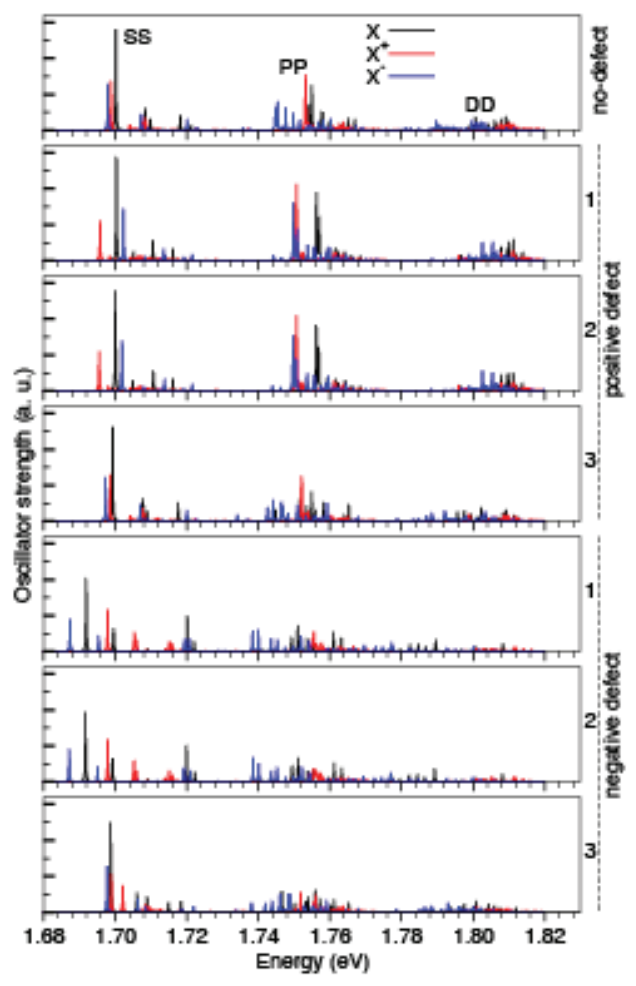

FIG. 6: Absorption spectra of neutral excitons (X) and charged excitons $\left(\mathrm{X}^{+}, \mathrm{X}^{-}\right)$in $\mathrm{GaAs} / \mathrm{Al}_{0.3} \mathrm{Ga}_{0.7}$ As QDs. The positions of the defects are defined $(1,2,3)$ in Fig 1 The labels SS, PP and DD refer to the transitions from the electron S, P, D states, respectively, to the hole states $\mathrm{h}_{0,1,2,3,4,5}$. Since the hole states have a heavily mixed orbital character (see Table III), transitions to all of them are possible, leading to a high multiplicity of peaks.

TABLE III: Decomposition of the atomistic hole wave functions in a GaAs QD into bulk $\Gamma$ point Bloch functions times envelope functions: $\Psi_{i}(\mathbf{r})=\sum_{n} \phi_{n}(\mathbf{r}) \psi_{n}(\mathbf{r})$ where $\phi_{n}$ is the Bloch function of band $n$ and $\psi_{n}$ is the corresponding envelope function. The decomposition factors tabulated here are the norm of the envelope functions for the three dominant valence bands $x, y$ and $z$. These bands are labelled by their axial angular momentum values $j=\frac{3}{2}$ for $\frac{(x+i y)}{\sqrt{2}} \uparrow, \frac{(x-i y)}{\sqrt{2}} \downarrow$; $j=\frac{1}{2}$ for $\frac{(x-i y)}{\sqrt{2}} \downarrow, \frac{(x+i y)}{\sqrt{2}} \uparrow$ and $j=\frac{1}{2}^{\prime}$ for $z \uparrow, z \downarrow$. Each envelope function contains a combination of angular momenta $(\mathrm{S}, \mathrm{P}, \mathrm{D})$, given in percent.

\begin{tabular}{c|rrr|rrr|rrr}
\hline \hline & \multicolumn{6}{|c}{ norm of the envelope function } \\
label & \multicolumn{3}{|c}{$j=\frac{3}{2}$} & \multicolumn{1}{c}{$j=\frac{1}{2}$} & \multicolumn{3}{c}{$j=\frac{1}{2}^{\prime}$} \\
& $\mathrm{S}$ & $\mathrm{P}$ & $\mathrm{D}$ & $\mathrm{S}$ & $\mathrm{P}$ & $\mathrm{D}$ & $\mathrm{S}$ & $\mathrm{P}$ & $\mathrm{D}$ \\
\hline $\mathrm{h}_{0}$ & $\mathbf{8 3 . 1}$ & 2.3 & 0.1 & 0.3 & 0.6 & 0.6 & 0.5 & 2.0 & 1.1 \\
$\mathrm{~h}_{1}$ & 3.6 & $\mathbf{4 0 . 2}$ & $\mathbf{6 . 4}$ & 4.5 & $\mathbf{7 . 4}$ & 0.4 & $\mathbf{8 . 2}$ & $\mathbf{1 3 . 6}$ & 0.7 \\
$\mathrm{~h}_{2}$ & 3.5 & $\mathbf{1 5 . 0}$ & $\mathbf{1 5 . 6}$ & $\mathbf{1 1 . 9}$ & 4.4 & 0.2 & $\mathbf{2 2 . 4}$ & $\mathbf{8 . 1}$ & 0.4 \\
$\mathrm{~h}_{3}$ & 1.5 & $\mathbf{5 2 . 5}$ & 3.0 & 0.2 & 4.2 & 1.1 & 0.4 & $\mathbf{7 . 2}$ & 2.9 \\
$\mathrm{~h}_{4}$ & $\mathbf{3 4 . 0}$ & $\mathbf{5 . 0}$ & 2.6 & 0.5 & 1.6 & $\mathbf{9 . 9}$ & 0.7 & 3.1 & $\mathbf{1 8 . 4}$ \\
$\mathrm{h}_{5}$ & $\mathbf{7 . 3}$ & $\mathbf{1 5 . 9}$ & $\mathbf{1 7 . 8}$ & 2.2 & $\mathbf{5 . 2}$ & 2.1 & 3.4 & $\mathbf{1 0 . 8}$ & 3.3 \\
\hline \hline
\end{tabular}

acter. This is a direct consequence of the fact that the
GaAs hole states have significant LH contributions due to the lack of strain in the structure (the LH band is closer to the HH band than in strained InAs dots). The hole states in GaAs QDs are even less "single-band"-like than in strained InGaAs QDs. The consequence of this twoband character of the hole states in GaAs is not obvious from the single-particle energies (see Fig [3) but very clear from the absorption spectra in Fig 6 . The single-band model for holes, that leads to the popular simplified optical selection rules - only SS, PP and DD transitions are allowed, leads to qualitatively wrong results. In GaAs, the mixed transitions "SP", "SD" where the hole is in a nominally $\mathrm{P}$ and D state are bright. Simply as a consequence, that these states have a significant $\mathrm{S}$ character, as given in Table III

The introduction of charged defects generally reduces the symmetry and introduces more LH-character in the hole states and more angular momentum mixing. As a consequence some transitions that were dark following the "single-band optical selection rules" are now bright. This can be seen especially for the InGaAs QD in Fig 5 that have relatively pure angular momentum hole states. In the defect free case, mainly the SS, PP and DD transitions are bright. The introduction of negative point charges leads to the appearance of nominally forbidden PS transitions. In the case of the GaAs QD the structure without defect has already a high level of angular momentum mixing (Table III) that the effect is quantitative which leads to the appearance of new peaks in the spectrum. We note that the oscillator strength for the SS transition is only slightly reduced in the case of the negative defects placed close to the center of the QDs (position 1 and 2), for other cases it remains nearly unchanged.

In summary, we investigate different point defect configurations in strained $\mathrm{In}_{0.6} \mathrm{Ga}_{0.4} \mathrm{As} / \mathrm{GaAs}$ and strain-free GaAs $/ \mathrm{Al}_{0.3} \mathrm{Ga}_{0.7}$ As QDs. Depending upon the relative position of the charged defects, we obtain significant modifications in the single particle electronic structure and excitonic spectra in both strained and strain-free QDs. However, the FSS, polarization anisotropy and polarization direction in strained QDs remain nearly unaffected, while in strain-free QDs these quantities change significantly. We furthermore highlight that the hole states in GaAs QDs deviate significantly from a singleband object. We find LH components of up to $20 \%$ and nominally "P" states can have up to $30 \%$ orbital S-character. This leads to a complex absorption picture with many peaks in GaAs QDs, compared to the case of strained InGaAs QDs.

\section{Acknowledgments}

We would like to acknowledge financial support by the BMBF (QuaHL-Rep, Contract No. 01BQ1034). 
1 E. G. Seebauer and M. C. Kratzer, Materials Science and Engineering: R: Reports 55, 57 (2006).

2 P. Michler, A. Kiraz, C. Becher, W. V. Schoenfeld, P. M. Petroff, L. D. Zhang, E. Hu, and A. Imamoglu, Science 290, 2282 (2000).

3 S. Cortez, O. Krebs, S. Laurent, M. Senes, X. Marie, P. Voisin, R. Ferreira, G. Bastard, J. M. Gerard, and T. Amand, Phys. Rev. Lett. 89, 207401 (2002).

${ }^{4}$ X. Michalet, F. F. Pinaud, L. A. Bentolila, J. M. Tsay, S. Doose, J. J. Li, G. Sundaresan, A. M. Wu, S. S. Gambhir, and S. Weiss, Science 307, 538 (2005).

5 B. D. Gerardot, D. Brunner, P. A. Dalgarno, P. Ohberg, S. Seidl, M. Kroner, K. Karrai, N. G. Stoltz, P. M. Petroff, and R. J. Warburton, Nature 451, 441 (2008).

6 R. B. Patel, A. J. Bennett, I. Farrer, C. A. Nicoll, D. A. Ritchie, and A. J. Shields, Nat. Photon. 4, 632 (2010).

7 M. Kroner, A. O. Govorov, S. Remi, B. Biedermann, S. Seidl, A. Badolato, P. M. Petroff, W. Zhang, R. Barbour, B. D. Gerardot, et al., Nature 451, 311 (2008).

8 C. Santori and Y. Yamamoto, Nat. Phys. 5, 173 (2009).

9 J.-F. Chen, R.-S. Hsiao, S.-H. Shih, P.-Y. Wang, J.-S. Wang, and J. Y. Chi, Jpn. J. Appl. Phys. 43, L1150 (2004).

10 D. G. Deppe, N. Holonyak, W. E. Plano, V. M. Robbins, J. M. Dallesasse, K. C. Hsieh, and J. E. Baker, J. Appl. Phys. 64, 1838 (1988).

11 X. Q. Meng, Z. Q. Chen, P. Jin, Z. G. Wang, and L. Wei, Appl. Phys. Lett. 91, 093510 (2007).

12 A. E. Belyaev, S. T. Stoddart, P. M. Martin, P. C. Main, L. Eaves, and M. Henini, Appl. Phys. Lett. 76, 3570 (2000).

13 J. Li, S.-H. Wei, and L.-W. Wang, Phys. Rev. Lett. 94, 185501 (2005).

14 T. Nguyen-Duc, V. L. Thanh, V. Yam, P. Boucaud, D. Bouchier, O. Schmidt, and J. Derrien, Thin Solid Films 508, 207 (2006).

15 G. N. Semenova, Y. F. Venger, M. Y. Valakh, Y. G. Sadofyev, N. O. Korsunska, V. V. Strelchuk, L. V. Borkovska, V. P. Papusha, and M. V. Vuychik, J. Phys.: Conden. Matter 14, 13375 (2002).

${ }^{16}$ H. Kamada and T. Kutsuwa, Phys. Rev. B 78, 155324
(2008).

17 A. L. Efros and M. Rosen, Phys. Rev. Lett. 78, 1110 (1997).

18 V. I. Klimov, A. A. Mikhailovsky, D. W. McBranch, C. A. Leatherdale, and M. G. Bawendi, Science 287, 1011 (2000).

19 B. Mahler, P. Spinicelli, S. Buil, X. Quelin, J.-P. Hermier, and B. Dubertret, Nat. Mater. 7, 659 (2008).

20 S. Seidl, B. D. Gerardot, P. A. Dalgarno, K. Kowalik, A. W. Holleitner, P. M. Petroff, K. Karrai, and R. J. Warburton, Physica E: Low-dimensional Systems and Nanostructures 40, 2153 (2008).

21 J. D. Plumhof, V. Křápek, L. Wang, A. Schliwa, D. Bimberg, A. Rastelli, and O. G. Schmidt, Phys. Rev. B 81, 121309 (2010).

${ }^{22}$ R. Seguin, A. Schliwa, S. Rodt, K. Potschke, U. W. Pohl, and D. Bimberg, Phys. Rev. Lett. 95, 257402 (2005).

23 C.-H. Lin, W.-T. You, H.-Y. Chou, S.-J. Cheng, S.-D. Lin, and W.-H. Chang, Phys. Rev. B 83, 075317 (2011).

24 N. Porras-Montenegro and S. T. Pérez-Merchancano, Phys. Rev. B 46, 9780 (1992).

25 S. T. Pantelides, Rev. Mod. Phys. 50, 797 (1978).

26 A. S. Martins, J. G. Menchero, R. B. Capaz, and B. Koiller, Phys. Rev. B 65, 245205 (2002).

27 P. N. Keating, Phys. Rev 145, 637 (1966).

28 A. J. Williamson, L.-W. Wang, and A. Zunger, Phys. Rev. B 62, 12963 (2000).

29 L.-W. Wang and A. Zunger, Phys. Rev. B 59, 15806 (1999).

30 G. Bester, S. Nair, and A. Zunger, Phys. Rev. B 67, 161306 (2003).

31 A. Franceschetti, H. Fu, L.-W. Wang, and A. Zunger, Phys. Rev. B 60, 1819 (1999).

32 R. Singh and G. Bester, Phys. Rev. Lett. 104, 196803 (2010).

33 A. J. Bennett, M. A. Pooley, R. M. Stevenson, M. B. Ward, R. B. Patel, A. B. de la Giroday, N. Skold, I. Farrer, C. A. Nicoll, D. A. Ritchie, et al., Nat. Phys. 6, 947 (2010). 